\title{
Tissue-specific down-regulation of S-adenosyl-homocysteine via suppression of dAhcyL1/dAhcyL2 extends health span and life span in Drosophila
}

\author{
Andrey A. Parkhitko, ${ }^{1}$ Richard Binari, ${ }^{1,2}$ Nannan Zhang, ${ }^{1,3}$ John M. Asara, ${ }^{4,5}$ Fabio Demontis, ${ }^{6}$ \\ and Norbert Perrimon ${ }^{1,2}$ \\ ${ }^{1}$ Department of Genetics, Harvard Medical School, Boston, Massachusetts 02115, USA; ${ }^{2}$ Howard Hughes Medical Institute, \\ Boston, Massachusetts 02115, USA ${ }^{3}$ MOE Key Laboratory of Protein Sciences, Department of Pharmacology, School of Medicine, \\ Tsinghua University, Beijing 100084, China; ${ }^{4}$ Division of Signal Transduction, Beth Israel Deaconess Medical Center, Harvard \\ Medical School, Boston, Massachusetts 02115, USA; ${ }^{5}$ Department of Medicine, Harvard Medical School, Boston, Massachusetts \\ 02115, USA; ${ }^{6}$ Department of Developmental Neurobiology, Division of Developmental Biology, St. Jude Children's Research \\ Hospital, Memphis, Tennessee 38105, USA
}

Aging is a risk factor for many human pathologies and is characterized by extensive metabolic changes. Using targeted high-throughput metabolite profiling in Drosophila melanogaster at different ages, we demonstrate that methionine metabolism changes strikingly during aging. Methionine generates the methyl donor S-adenosylmethionine (SAM), which is converted via methylation to S-adenosyl-homocysteine (SAH), which accumulates during aging. A targeted RNAi screen against methionine pathway components revealed significant life span extension in response to down-regulation of two noncanonical Drosophila homologs of the SAH hydrolase Ahcy (S-adenosyl-L-homocysteine hydrolase [SAHH[), CG9977/dAhcyL1 and Ahcy89E/CG8956/dAhcyL2, which act as dominant-negative regulators of canonical AHCY. Importantly, tissue-specific down-regulation of dAhcyL1/L2 in the brain and intestine extends health and life span. Furthermore, metabolomic analysis of dAhcyL1-deficient flies revealed its effect on age-dependent metabolic reprogramming and H3K4 methylation. Altogether, reprogramming of methionine metabolism in young flies and suppression of age-dependent SAH accumulation lead to increased life span. These studies highlight the role of noncanonical Ahcy enzymes as determinants of healthy aging and longevity.

[Keywords: CG9977/AhcyL1; CG8956/Ahcy89E/AhcyL2; methionine restriction; aging; life span; S-adenosylhomocysteine $(\mathrm{SAH})]$

Supplemental material is available for this article.

Received April 5, 2016; revised version accepted May 17, 2016.

Aging is the primary risk factor for many major human pathologies, including cancer, diabetes, cardiovascular disorders, and neurodegenerative diseases (Lopez-Otin et al. 2013). Previous studies of the transcriptional changes that occur during Drosophila aging have revealed that genes encoding members of metabolic pathways are among the most affected (Landis et al. 2004; Lai et al. 2007). In addition, analyses of changes associated with dietary restriction (DR) that slows down the aging process have also demonstrated dramatic changes in the expression of different metabolic genes (Pletcher et al. 2002). Similarly, studies in worms (Fuchs et al. 2010), mice

Corresponding authors: aparkhitko@genetics.med.harvard.edu, perrimon@receptor.med.harvard.edu

Article published online ahead of print. Article and publication date are online at http://www.genesdev.org/cgi/doi/10.1101/gad.282277.116.
(Tomas-Loba et al. 2013), and humans (Yu et al. 2012) have documented changes in the metabolome during the aging process. Recently, untargeted metabolomics analysis in flies (Hoffman et al. 2014) has suggested that DR might reverse age-dependent metabolic reprogramming at the tissue (Laye et al. 2015) and whole-organism (Avanesov et al. 2014) levels. Despite these studies, the mechanisms underlying age-dependent metabolic reprogramming, the nature of the metabolites that change with time, and their effect on life span are still poorly characterized.

(C) 2016 Parkhitko et al. This article is distributed exclusively by Cold Spring Harbor Laboratory Press for the first six months after the full-issue publication date (see http://genesdev.cshlp.org/site/misc/terms.xhtml). After six months, it is available under a Creative Commons License (Attribution-NonCommercial 4.0 International), as described at http:// creativecommons.org/licenses/by-nc/4.0/. 
A number of alterations in metabolic pathway activities are known to extend life span in flies and other organisms. Among them, perturbation of components of the mitochondrial respiratory complexes I, III, IV, and V (Copeland et al. 2009; Owusu-Ansah et al. 2013); increased mitochondrial uncoupling via expression of human UCP2 (Fridell et al. 2005); heterozygous mutations of AMP biosynthetic enzymes (Stenesen et al. 2013); reduced levels of Enigma, the enzyme responsible for $\beta$-oxidation of fatty acids (Mourikis et al. 2006); and reduced levels of Indy, which functions as a cation-independent electroneutral transporter for a variety of tricarboxylic acid cycle intermediates (Rogina et al. 2000) extend life span in Drosophila. In addition, key longevity regulators such as insulin receptor substrate (IRS)/chico and JNK are known to reprogram whole-body metabolism, but it is unknown whether this reprogramming is responsible for life span extension (Clancy et al. 2001; Wang et al. 2003).

Another level of complexity between metabolism and aging arises from the observation that different tissues have different metabolic requirements and that alterations of different metabolic components or upstream regulators of metabolism in one tissue can affect aging of other tissues and life span (Finkel 2015). For example, muscle-specific FOXO/4E-BP signaling retards muscle aging in Drosophila in a cell-autonomous manner and nonautonomously extends life span and preserves proteostasis in other aging tissues such as the brain, the retina, and adipose tissue (Demontis and Perrimon 2010). Similarly, overexpression of AMPK in the adult Drosophila nervous system nonautonomously maintains proteostasis during muscle aging and extends organismal life span (Ulgherait et al. 2014). In addition, muscle-specific mitochondrial injury promotes organismal life span via activation of mtUPR and increased production of ImpL2, an insulin growth factorbinding protein (IGFBP)-like protein (Owusu-Ansah et al. 2013). Moreover, muscle-specific expression of the transcription factor Mnt extends life span by reducing ribosome biogenesis and promoting the expression of the myokine Myoglianin (Demontis et al. 2014).

To expand our knowledge of the regulation of life span by metabolism, we performed high-throughput metabolite profiling of Drosophila melanogaster to identify changes that may correlate with aging. Strikingly, methionine metabolism emerged as one of the most regulated metabolic pathways with age. To test the role of the methionine pathway in life span determination, we performed a targeted RNAi screen against most of the methionine pathway components and related enzymes. Unexpectedly, ubiquitous down-regulation of two Drosophila homologs of S-adenosyl-homocysteine (SAH) hydrolase-like proteins, CG9977/dAhcyL1 (S-adenosyl-L-homocysteine hydrolase [SAHH]) and CG8956/Ahcy89E/dAhcyL2, significantly extended life span. Moreover, brain-specific down-regulation of $d A h c y L 1$ and intestine-specific down-regulation of both $d A h c y L 1$ and $d A h c y L 2$ increased life span. Importantly, down-regulation of $d A h c y L 1$ extended not only life span but also health span. Finally, suppression of $d A h c y L 1$ activities decreased the level of $\mathrm{SAH}$, as determined by tandem mass spectrometry (MS/
MS), and suppressed H3K4 trimethylation (H3K4me3), thus phenocopying methionine starvation. Altogether, our data demonstrate that dAhcyL1 and dAhcyL2 encode new key regulators of age-dependent metabolic reprogramming and control both health span and life span.

\section{Results \\ Age-dependent reprogramming of methionine metabolism}

To investigate the impact of age on the metabolism of adult flies, we performed high-throughput steady-state metabolite profiling using targeted liquid chromatography-MS/MS (LC-MS/MS) (Yuan et al. 2012) of two common Drosophila strains (yw and OregonR [OreR]) at 1, 4, and 7 wk of age (Fig. 1A). We identified 107 and 112 metabolites that were significantly changed between 1 and $7 \mathrm{wk}$ in OreR males and yw males, respectively, with 51 changed in both strains (Fig. 1B,C). A similar analysis in females identified 121 and 113 metabolites that were significantly affected, with 54 in common (Supplemental Fig. 1A,B). Interestingly, only 21 regulated metabolites were shared between males and females, indicating that sex has a strong effect on age-specific variations of the metabolome (Supplemental Fig. 2E and List 1). Strikingly, among the 21 metabolites that similarly changed with age in both males and females of different fly strains, five belong to the methionine pathway (betaine, cystathionine, spermidine, methionine sulfoxide, and glutathione) (Fig. 1D,E). Next, we focused our analysis on males, as metabolic changes in females could be affected by egg production, which decreases with age. Principal component analysis (PCA) of the measured metabolites clearly distinguished flies of different ages $(1,4$, and 7 wk of age) for both the OreR and yw strains (Supplemental Fig. 2F). Interestingly, the changes in metabolite concentrations were bidirectional in that about half of them were increased with age, whereas others were decreased, suggesting that the metabolic changes observed during aging did not reflect simply a decrease in metabolic activity but rather an age-dependent metabolic reprogramming. Although we measured steady-state metabolite levels (Yuan et al. 2012), mapping the metabolites onto the methionine metabolism pathway (Fig. 1F) suggested a decreased activity of the methionine cycle with age. In the methionine cycle, S-adenosylmethionine (SAM) is used for methylation reactions and generates $\mathrm{SAH}$, which is further hydrolyzed by AHCY into homocysteine and adenosine (Brosnan and Brosnan 2006). Homocysteine is a key branch point intermediate in the methionine cycle, as it can be remethylated back to methionine using betaine or 5-methyl-THF as methyl donors and retained in the methionine cycle. Alternatively, homocysteine can be converted into cysteine, the precursor of glutathione, via generation of cystathionine (Fig. 1F; Brosnan and Brosnan 2006). Strikingly, the levels of methionine, cystathionine, and glutathione were decreased with age, whereas the levels of betaine as well as $\mathrm{SAH}$ were increased, suggesting that methionine 


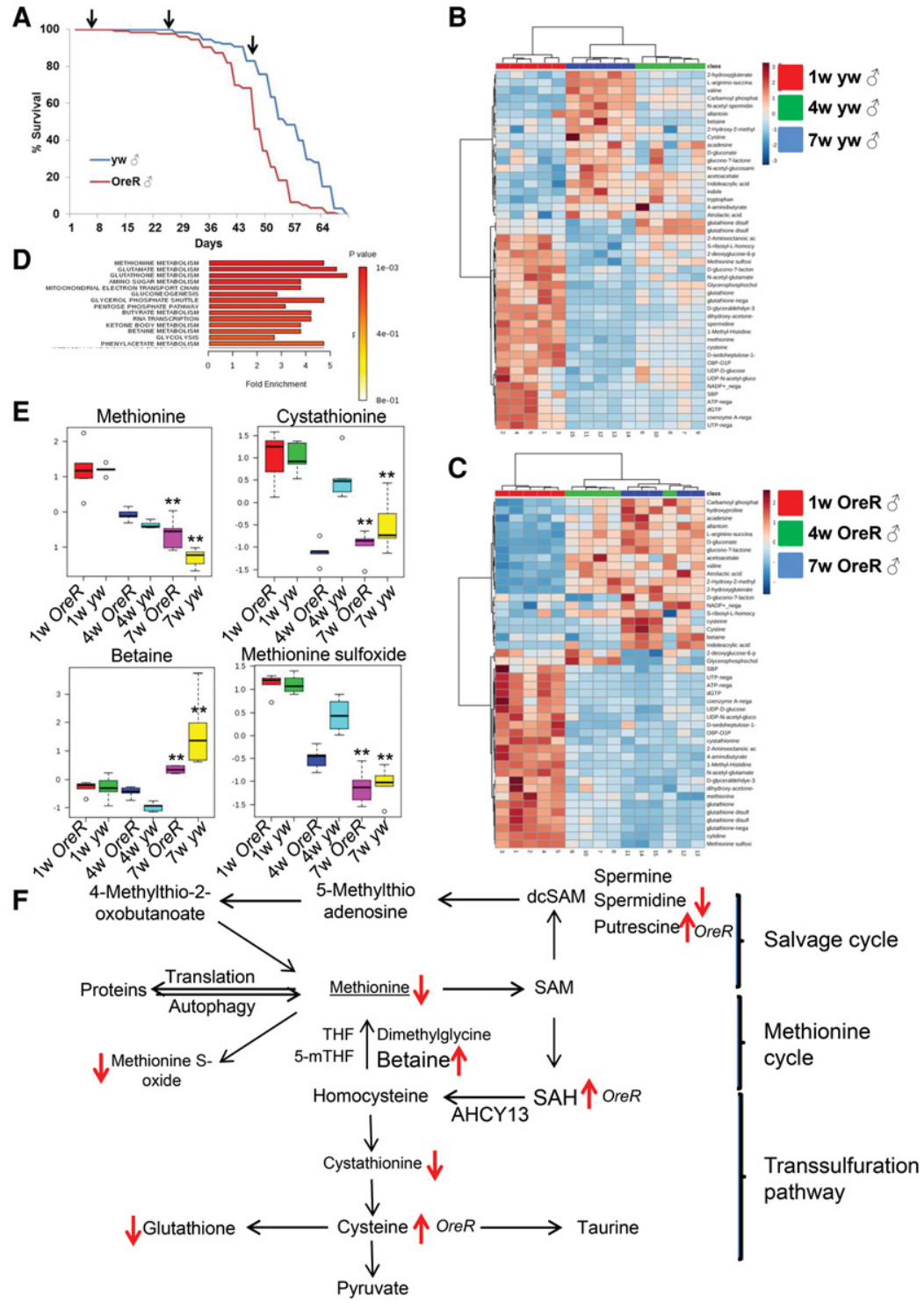

Figure 1. Age-dependent metabolism reprogramming. (A) Life spans of male yw (blue) and OreR (red) flies. Samples were collected at 1, 4, and 7 wk of age for metabolomics analyses by LC-MS/ MS. $(B, C)$ Heat maps showing the metabolites significantly affected in both $y w$ and OreR flies between 1 and 7 wk of age. $(D)$ Metabolic set enrichment analysis of the metabolites that changed significantly in both $y w$ and OreR flies between 1 and 7 wk of age. (E) Box plots of individual methionine metabolism pathway metabolites significantly affected in both $y w$ and OreR flies between 1 and 7 wk of age. $(F)$ Methionine metabolism pathway. Arrows indicate the metabolites significantly affected in $y w$ and OreR flies. metabolism was decreased with age. Our metabolomics results suggest that decreased activity of the methionine cycle results in a decreased demand for betaine and is associated with the suppressed clearance of SAH. Altogether, these findings suggest that whole-body methionine metabolism is reprogrammed during aging, which is accompanied by $\mathrm{SAH}$ accumulation.

\section{Naturally selected long-lived flies have altered methionine metabolism}

To test whether increased life span is associated with specific changes in methionine metabolism, we compared the metabolomes of long-lived flies with wild type. To prevent bias for specific signaling pathways associated with life span extension, we used flies with increased longevity that have been selected for delayed reproductive senescence. These long-lived flies were selected for over 170 generations and maintained on a generation interval of $70 \mathrm{~d}$, while unselected control lines were maintained on a 2-wk generation interval (Carnes et al. 2015). We compared the metabolic profiles of males of two different long-lived lines, $\mathrm{O} 1$ and $\mathrm{O} 3$, with a control line (B3) at 1 wk of age (Fig. 2A). PCA clearly distinguished control and long-lived flies when $100 \%$ of all flies were still alive (Fig. 2B). Strikingly, we found dramatic differences between control B3 and long-lived $\mathrm{O} 1$ and $\mathrm{O} 3$ flies for many metabolites associated with methionine metabolism (betaine, spermidine, methionine, and methionine sulfoxide), including those that changed with age (Fig. 2C,D). However, contrary to the changes observed with age, the changes in steady-state metabolite levels in $\mathrm{O} 1$ and $\mathrm{O} 3$ flies suggested an increased flux via the methionine cycle. Specifically, the lower levels of betaine and 5-methyl-THF in $\mathrm{O} 1$ and $\mathrm{O} 3$ flies suggested increased demands for methyl donors in the methionine cycle for methionine production. In addition, $\mathrm{O} 1$ and $\mathrm{O} 3$ flies had lower levels of homocysteine, suggesting that this 
A

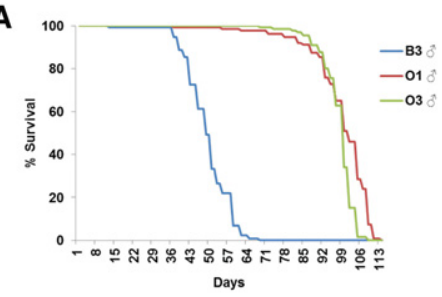

C

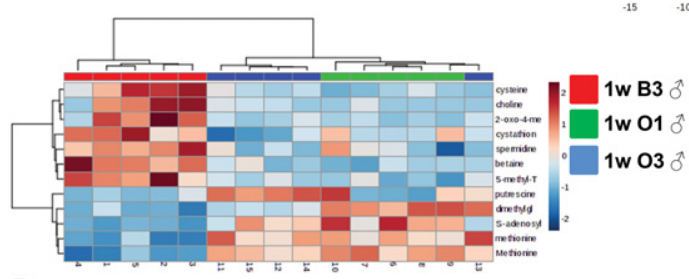

D

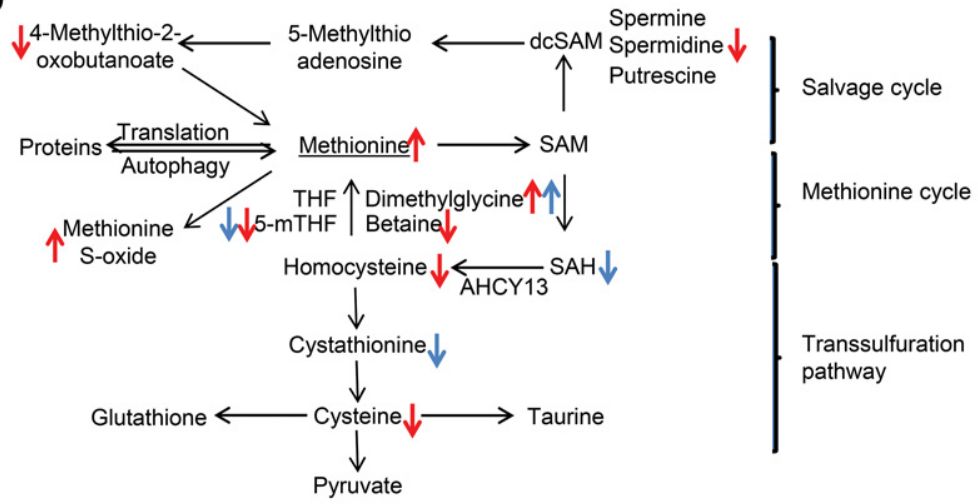

B

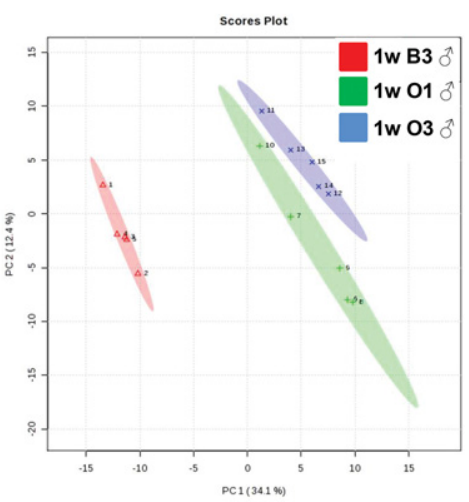

Figure 2. Methionine metabolism reprogramming in naturally selected long-lived flies. $(A)$ Life spans of control (B3) and long-lived (O1 and $\mathrm{O} 3$ ) males. $(B)$ $\mathrm{PCA}$ of control (B3) and long-lived (O1 and O3) males. $(C)$ Heat map showing the metabolites of the methionine metabolism pathway. (D) The methionine metabolism pathway. Arrows point to the metabolites that are significantly changed between control (B3) and long-lived (O1 and O3) flies at 1 wk of age (red) and $4 \mathrm{wk}$ of age (blue).

V $01 / 03$ vs B3 1w

VI 01/03 vs B37w

metabolite is more efficiently transformed back into methionine. In the methionine cycle, homocysteine can be irreversibly converted into cystathionine and cysteine and then into glutathione and taurine (major antioxidants) via the transsulfuration branch (Brosnan and Brosnan 2006). Interestingly, long-lived O1 and O3 flies had lower levels of cystathionine and cysteine compared with B3 control flies, suggesting that the activity of the transsulfuration branch is increased in $\mathrm{B} 3$ flies. The increased activity of the transsulfuration branch in control B3 flies could be associated with increased demands for antioxidant molecules due to increased requirements to cope with oxidative stress and the shunting of homocysteine away from the methionine cycle. In contrast, the higher activity of the methionine cycle in long-lived $\mathrm{O} 1$ and $\mathrm{O} 3$ flies would allow better clearance of its intermediate metabolites. Consistently, the levels of SAH were significantly decreased at $4 \mathrm{wk}$ of age in long-lived $\mathrm{O} 1$ and $\mathrm{O} 3$ flies compared with control B3 flies. In addition and in agreement with the potentially higher activity of the methionine cycle in $\mathrm{O} 1$ and $\mathrm{O} 3$ flies, the level of methionine was sig- nificantly higher in long-lived flies at $1 \mathrm{wk}$ of age (Fig. 2C). These results indicate that the high level of methionine itself is not associated with decreased life span and suggest that the rate of methionine processing is important for life span. Altogether, our data support the model that reprogramming of methionine metabolism in young flies and suppression of age-dependent SAH accumulation is associated with increased life span.

\section{dAhcyL1 and dAhcyL2 regulate both life span and health span}

Because methionine restriction extends life span in different organisms (Orentreich et al. 1993; Koziel et al. 2014; Lee et al. 2014), multiple components of methionine metabolism change with age, and methionine levels are significantly different in short- and long-lived flies, we systematically evaluated the effect of methionine metabolism enzymes on life span using RNAi. To avoid lethality during development and differences in genetic background, we used the Actin GeneSwitch (ActinGS)- 
inducible Gal4/UAS expression system /Osterwalder et al. 2001; Roman et al. 2001), by which UAS-RNAi expression is driven by Gal4 when flies are fed mifepristone (RU486). As expected, RNAi against white $(w)$ or luciferase overexpression did not affect life span, whereas Rheb RNAi significantly extended life span $(12.9 \%$ median life span increase, $P<0.0001$ ) (Fig. 3A,B). Rheb was used as a control, as it encodes a positive regulator of mTOR activity, and mTOR suppression has been shown to extend life span in different organisms, including Drosophila (Bjedov et al. 2010). The methionine metabolism pathway consists of three branches (salvage, de novo, and transsulfuration pathways), and we used 53 different RNAi and overexpression lines to target 35 enzymes in all branches of methionine metabolism and adjacent pathways (Supplemental Table 1). As reported previously (Kabil et al. 2011), down-regulation of cystathionine $\beta$-synthase (CBS), a rate-limiting enzyme in the transsulfuration pathway responsible for the production of glutathione and taurine, led to shortened life span (16\% decrease in median life span, $P<0.0001$ ) (Fig. 3A). In addition, down-regulation of methionyl-tRNA synthetase/ CG15100 (which covalently links methionine with its cognate tRNA) and RNAi against the mitochondrial SAM transporter CG4743 exhibited the most dramatic life span decreases $(25 \%$ and $34 \%$ decrease in median life span, respectively) (Fig. 3A). Unexpectedly, down-regulation of two out of the three Drosophila homologs of

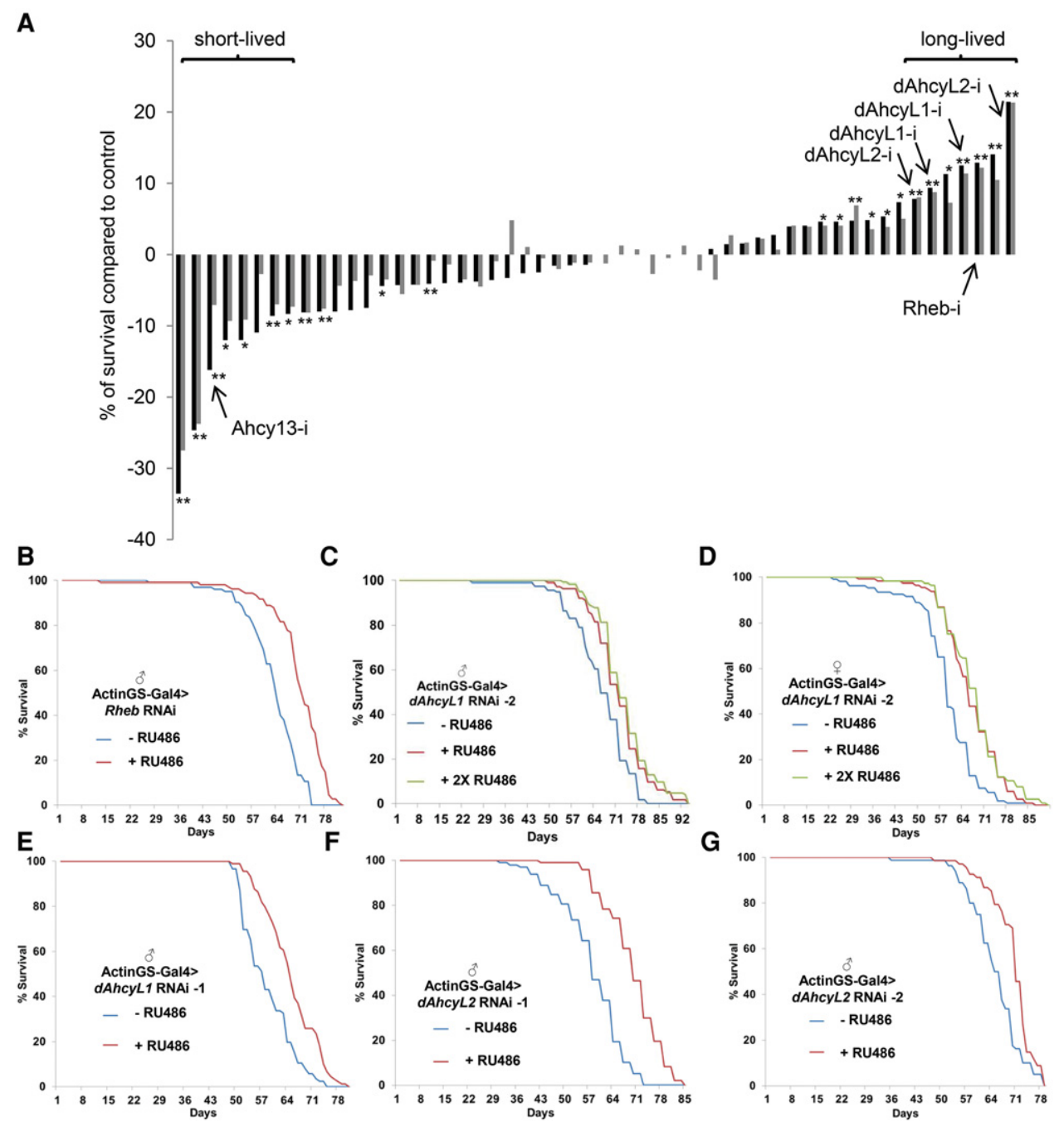

Figure 3. Screen of methionine metabolism components for life span regulation. $(A)$ RNAi screen results ordered by ascending life span. Each bar represents the difference ([black bars] median; [gray bars] mean) in life span between ActinGS $>$ RNAi flies and ActinGS $>$ RNAi flies fed with RU486 starting at day 7. $\left(^{*}\right) P<0.05$; $\left(^{* *}\right) P<0.01$. $(B)$ Ubiquitous adult-onset expression of Rheb RNAi increases life span in males. $P<0.0001$. (C) Ubiquitous adult-onset expression of $d A$ hcyL1 RNAi increases life span in males. $P<0.0001$ (for both $1 \times$ and $2 \times$ concentrations of RU486). (D) Ubiquitous adult-onset expression of $d A$ hcyL1 RNAi-2 increases life span in females. $P<0.0001$ (for both $1 \times$ and $2 \times$ concentrations of RU486). (E) Ubiquitous adult-onset expression of dAhcyL1 RNAi-1 (HM05009) increases life span in males. $P<0.0001$. $(F)$ Ubiquitous adult-onset expression of $d A$ hcyL2 RNAi-1 increases life span in males. $P<0.0001$. (G) Ubiquitous adult-onset expression of $d A h c y L 2$ RNAi-2 increases life span in males. $P<0.0001$. 
AHCY-dAhcyL1 (dAhcyL1 RNAi-2, 9.4\% median life span increase in males and $11 \%$ in females [Fig. 3C,D]; and $d A h c y L 1$ RNAi-1, $12.5 \%$ median life span increase, $P<0.0001$ [Fig. 3E]) and dAhcyL2 (dAhcyL2 RNAi-1, $21.4 \%$ median life span increase, $P<0.0001$ [Fig. 3F]; and $d A$ hcyL2 RNAi-2, $7.8 \%$ median life span increase, $P$ $<0.0001$ [Fig. 3G]) - significantly extended life span in adult males and females. The knockdown efficacy of dAhcyL1 (Supplemental Fig. 3A) and dAhcyL2 (Supplemental Fig. 3B) RNAi lines was tested using the ubiquitous Actin5c-Gal4 driver, and stronger levels of RNAi efficiency were consistent with more robust life span extension. Importantly, the level of $d A h c y L 1$ was significantly decreased in long-lived $\mathrm{O} 1$ and $\mathrm{O} 3$ flies compared with B3 control flies at 1 wk of age, which is consistent with low levels of dAhcyL1 increasing life span (Supplemental Fig. 3C). However, these differences are only correlative, and there are multiple changes that explain the increased life span of long-lived $\mathrm{O} 1$ and $\mathrm{O} 3$ flies. In summary, our data suggest that dAhcyL1 and dAhcyL2 are new life span regulators.

\section{dAhcyL1 and dAhcyL2 regulate health span}

To characterize how loss of $d A h c y L 1$ and $d A h c y L 2$ affects health span, we tested their abilities to delay age-dependent tissue functional decline as measured by maintaining climbing activity (indicative of neuromuscular function), intestine barrier, and egg laying. Normally, skeletal muscle function declines during aging in flies (Demontis et al. 2013). However, down-regulation of $d A h c y L 1$ (Fig. 4A,B) and dAhcyL2 (Fig. 4C) suppressed the age-dependent decrease of climbing activity, as determined by negative geotaxis assays. Aging is also characterized by decreased reproductive function, which, by itself, can extend life span (Sgro and Partridge 1999). However, down-regulation of dAhcyL1 increased fecundity (Fig. 4D), suggesting that dAhcyL1-increased life span is not due to impaired reproduction. Finally, we measured the intestinal integrity of flies fed with a nonabsorbable blue food dye (the "Smurf assay") (Rera et al. 2011). Strikingly, down-regulation of both dAhcyL1 (Fig. 4E) and dAhcyL2 (Fig. 4F) retarded the age-related onset of the "Smurf" phenotype, suggesting improved intestinal integrity in old flies. As expected, expression of control $(w)$ RNAi did not affect egg production, climbing, or intestinal integrity (data not shown). An additional hallmark of extended longevity is an increased ability to withstand oxidative stress. Thus, we tested the survival of ActinGS-Gal4>dAhcyL1 RNAi-1 flies with and without RU486 added to food containing $10 \mathrm{mM}$ methyl viologen dichloride hydrate (paraquat), an oxidant compound. Interestingly, we observed no difference in the survival of control and dAhcyL1 RNAi flies (Supplemental Fig. 4), suggesting that the life span increase associated with down-regulation of dAhcyL1 is not caused by an increased ability to withstand oxidative stress. In summary, our data indicate that dAhcyL1 and dAhcyL2 encode new life span and health span regulators and that their effects are independent of oxidative stress resistance.
Down-regulation of dAhcyL1 in the brain and dAhcyL1 and $d A h c y L 2$ in the intestine increase life span

To further dissect the role of $d A h c y L 1$ and $d A h c y L 2$ in life span, we tested whether specific tissues were responsible for life span extension. Expression of either dAhcyL1 RNAi-1 or dAhcyL2 RNAi-1 in the whole-body fat body of adult flies starting at 1 wk of age using the GeneSwitch driver strain (WB-FB-GS), which contains both a head fat body driver (S1-32) and a body fat body driver (S1-106) (Giannakou et al. 2007; Shen et al. 2009), did not affect life span in either males or females (Fig. 4G,H). However, using the Elav GeneSwitch driver (Elav-GS), which drives nervous system-specific expression (Osterwalder et al. 2001; Shen et al. 2009), a significant extension of life span was observed when $d A h c y L 1$ RNAi-1 was expressed starting at $1 \mathrm{wk}$ in both males and females. A similar experiment with $d A h c y L 2$ RNAi failed to reveal an effect (Fig. $4 \mathrm{I}, \mathrm{J})$. In addition, the TIGS-2 GeneSwitch driver (TIGS-2), which is associated with digestive tract-specific expression (Poirier et al. 2008; Rera et al. 2011), significantly extended life span when either $d A h c y L 1$ RNAi-1 or $d A h c y L 2$ RNAi1 was expressed in adult females starting at 1 wk of age (Fig. $4 \mathrm{~K}, \mathrm{~L}$ ) - a result consistent with the observation that downregulation of both $d A h c y L 1$ and $d A h c y L 2$ suppressed agedependent loss of intestine barrier function (Fig. 4E,F). As expected, expression of control (w) RNAi did not affect life span with any organ-specific GeneSwitch driver (data not shown). Altogether, our results suggest that tissue-specific down-regulation of both $d A h c y L 1$ and $d A h c y L 2$ is sufficient to extend life span.

\section{Noncanonical functions of $\mathrm{AAHCY}$ proteins are not involved in life span regulation}

Canonical AHCY hydrolyzes SAH to adenosine and homocysteine. While adenosine is further deaminated to inosine, homocysteine is either remethylated to methionine or enters the transsulfuration pathway. Due to critical mutations in their AHCY domains, dAHCYL1 and dAHCYL2 proteins most likely have lost their canonical enzymatic functions. However, via heteromultimerization, ACHYL can suppress the enzymatic activity of AHCY and thus act as a dominant-negative regulator of canonical AHCY (Devogelaere et al. 2008). In addition, dAHCYL1 and dAHCYL2 proteins contain N-terminal IRBIT domains, and dAHCYL2 contains an N-terminal $\mathrm{P} / \mathrm{A}$ domain enriched in Pro/Ala residues (Devogelaere et al. 2008), which give dAHCYL1/dAHCYL2 proteins AHCY-independent/noncanonical functions. Thus, we tested whether these AHCY-independent functions are responsible for the effect on life span. AHCYL1 functions as an inhibitor of $\mathrm{IP}_{3} \mathrm{R}$, which is a critical regulator of intracellular $\mathrm{Ca}^{2+}$ signaling (Ando et al. 2006). Binding of $\mathrm{IP}_{3}$ to $\mathrm{IP}_{3} \mathrm{R}$ causes the opening of the $\mathrm{IP}_{3} \mathrm{R}$ channel and $\mathrm{Ca}^{2+}$ release, whereas AHCYL1 competes with $\mathrm{IP}_{3}$ for $\mathrm{IP}_{3} \mathrm{R}$ binding but is unable to open the $\mathrm{IP}_{3} \mathrm{R}$ channel (Fig. 5A; Ando et al. 2006). Life span was not affected by either down-regulation (Itp-r83A RNAi) (Fig. 5B) or up-regulation (UAS-Itp-r83A) (Fig. 5C) of $\mathrm{IP}_{3} \mathrm{R}$, suggesting that life 
A
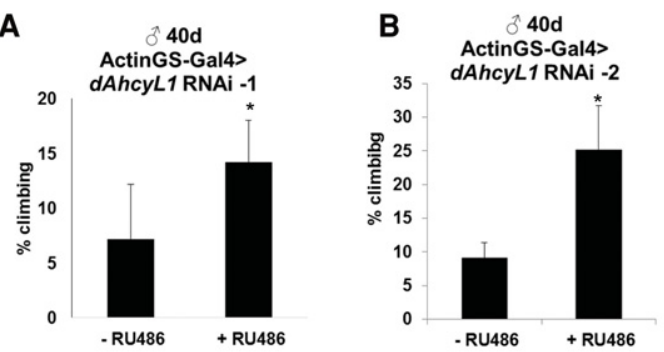

D

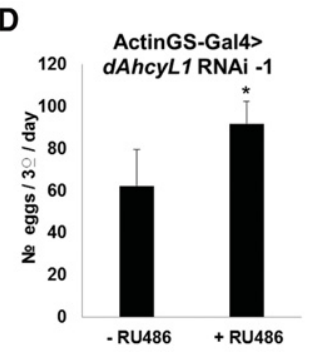

G

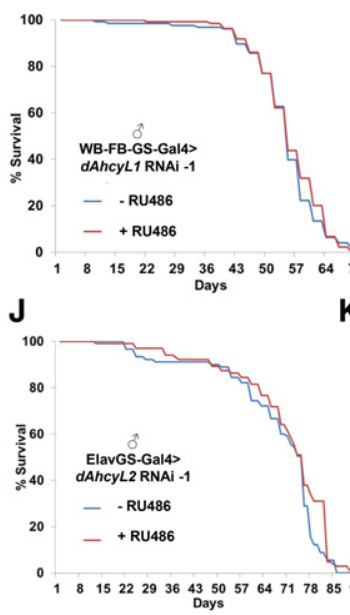

E

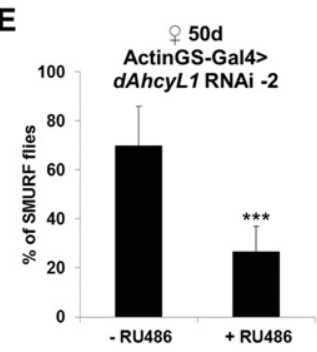

H
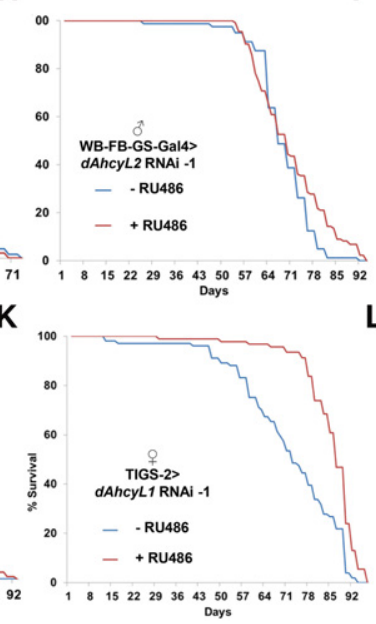

I
C

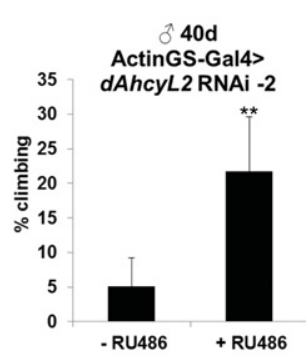

$\mathbf{F}$
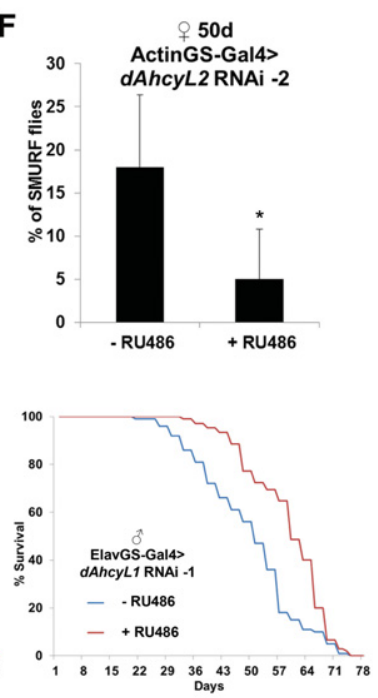

$\mathbf{L}_{10}$

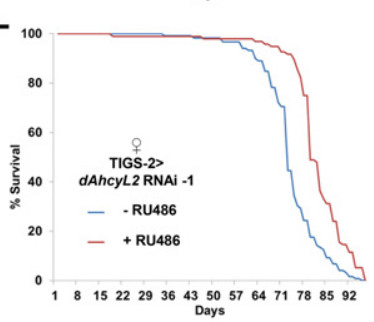

Figure 4. $d A h c y L 1$ and $d A h c y L 2$ regulate health span. $(A)$ Ubiquitous adult-onset expression of $d A h c y L 1$ RNAi-1 improves climbing ability in 40-d-old males. $\left({ }^{*}\right) P<0.05$. Means \pm SD. (B) Ubiquitous adult-onset expression of dAhcyL1 RNAi-2 improves climbing ability in 40-d-old males. $\left({ }^{*}\right) P<0.05$. Means \pm SD. $(C)$ Ubiquitous adult-onset expression of $d A h c y L 2$ RNAi-1 improves climbing ability in 40-d-old males. $\left.{ }^{* *}\right) P<0.01$. Means \pm SD. $(D)$ Ubiquitous adult-onset expression of dAhcyL1 RNAi-1 improves egg laying in 40-d-old flies. $\left({ }^{*}\right) P<$ 0.05 . Means \pm SD. $(E)$ Ubiquitous adult-onset expression of $d A$ hcyL1 RNAi-2 suppresses the number of "Smurf" females at $40 \mathrm{~d}$ of age. ${ }^{(* * *)} P<0.001$. Means \pm SD. $(F)$ Ubiquitous adult-onset expression of $d A h c y L 2$ RNAi-1 suppresses the number of "Smurf" females at $40 \mathrm{~d}$ of age. $\left({ }^{*}\right) P<0.05$. Means \pm SD. $(G)$ Fat body-specific adult-onset expression of dAhcyL1 RNAi-1 does not affect life span in males. (H) Fat body-specific adult-onset expression of dAhcyL2 RNAi-1 does not affect life span in males. (I) Brain-specific adult-onset expression of $d A h c y L 1$ RNAi-1 increases life span in males. $P<0.0001$. (J) Brain-specific adult-onset expression of dAhcyL2 RNAi-1 does not affect life span in males. $(K)$ Intestine-specific adult-onset expression of dAhcyL1 RNAi-1 increases life span in females. $\quad P<0.0001$. $\quad(L) \quad$ Intestine-specific adult-onset expression of $d A h c y L 1$ RNAi-2 increases life span in females. $P<0.001$ span extension caused by down-regulation of dAHCYL1 is independent of $\mathrm{IP}_{3} \mathrm{R}$. Note that the knockdown efficacy of the Itp-r83A RNAi line was tested using the ubiquitous Actin5c-Gal4 driver (Supplemental Fig. 3D).

IRBIT domain-containing proteins form a deoxyadenosine triphosphate (dATP)-dependent complex with ribonucleotide reductase (RNR), stabilizing dATP in the active site of RNR and thus inhibiting the enzyme (Fig. 5D; Arnaoutov and Dasso 2014). To test whether loss of dAHCYL1/dAHCYL2 activates RNR, we performed metabolomic profiling of flies expressing either $d A h c y L 1$ RNAi-1 or control RNAi (against $g f p$ ) using the ubiquitous temperature-sensitive (tubulin-Gal4, tubulin-Gal80ts) driver for $7 \mathrm{~d}$. No significant differences in dCDP, dTDP, dGDP, dTTP, and dGTP levels were observed between control and $d A h c y L 1$ RNAi-1 flies (Fig. 5E,F,G). Furthermore, as RNR is composed of two large (RnrL) and two small (RnrS) subunits that form an active tetramer (Fig. $5 \mathrm{D})$, activation of RNR would require coexpression of both subunits, and their overexpression would not necessarily increase RNR activity. To examine the role of RNR in life span regulation, we tested whether down-regulation of the large RNR subunit RnrL would affect life span. RnrL RNAi expression using the ActinGS system at 1 wk of age had no effect on life span in males (Fig. $5 \mathrm{H})$, suggesting that increased life span caused by dAHCYL1/dAHCYL2 down-regulation is unlikely linked to alteration in RNR activity. Note that the knockdown efficacy of the RnrL RNAi line was tested using Actin5c-Gal4 (Supplemental Fig. 3E). Altogether, our data suggest that the putative noncanonical functions of dAHCYL1/dAHCYL2 are unlikely to be connected to their regulation of life span.

dAhcyL1 and dAhcyL2 are involved in the regulation of methionine metabolism

As dAHCYL1 and dAHCYL2 proteins have most likely lost their enzyme activities, we tested whether they can affect the activity of canonical Ahcy13 via heteromultimerization (Fig. 6A). We confirmed the interaction between dAHCYL1 and Ahcy13 by coimmunoprecipitation in 
A

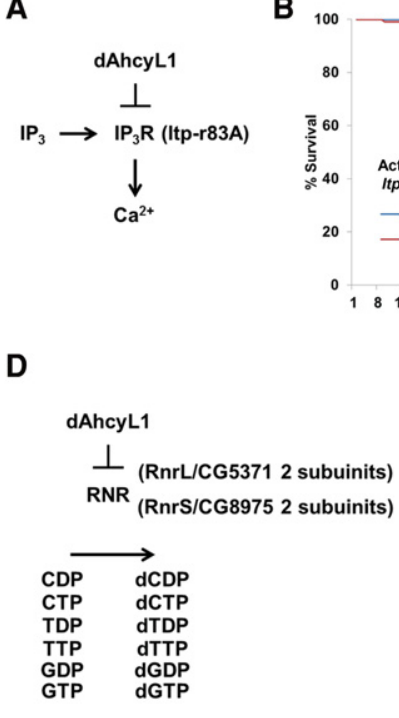

G

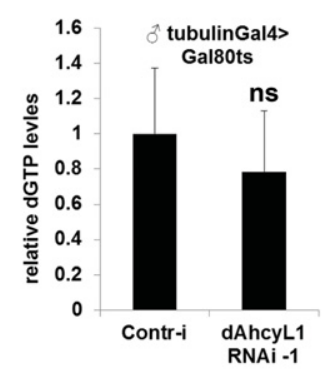

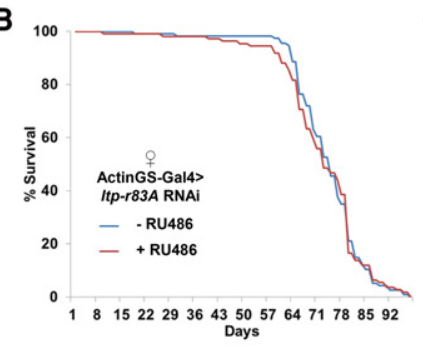

E

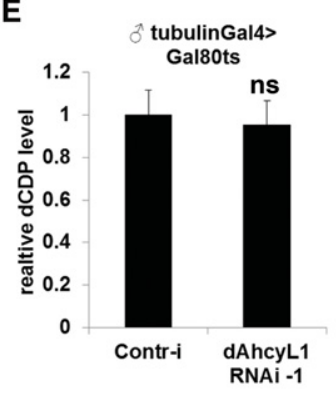

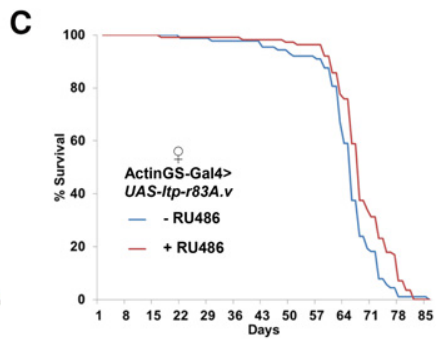

F

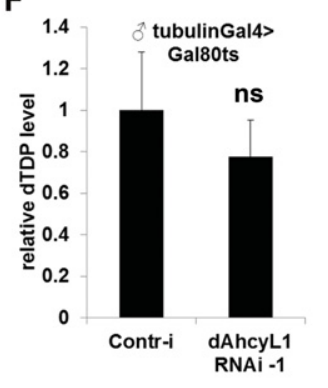

Figure 5. Noncanonical functions of dAhcy are not involved in life span regulation. (A) Scheme of IP ${ }_{3} \mathrm{R}-\mathrm{dAhcyL} 1$ function. (B) Ubiquitous adult-onset expression of Itp-r83A RNAi does not affect life span in females. $(C)$ Ubiquitous adult-onset expression of Itp-r83A.v does not affect life span in females. $(D)$ Scheme of ribonucleotide reductase (RNR) regulation by dAhcyL1. (E) Relative levels of dCDP in tubulinGal80ts, tubulinGal4 flies expressing either control $(g f p)$ or dAhcyL1 RNAi-1 for 2 wk at $29.5^{\circ} \mathrm{C}$. Means \pm SD. $(F)$ Relative levels of dTDP in tubulinGal80ts, tubulinGal4 flies expressing either control $(g f p)$ or dAhcyL1 RNAi-1 for 2 wk at $29.5^{\circ} \mathrm{C}$. Means \pm SD. $(G)$ Relative levels of dGTP in tubulinGal80ts, tubulinGal4 flies expressing either control $(g f p)$ or $d A h c y L 1$ RNAi- 1 for 2 wk at $29.5^{\circ} \mathrm{C}$. (H) Ubiquitous adult-onset expression of RnrL RNAi does not affect life span in males.

H

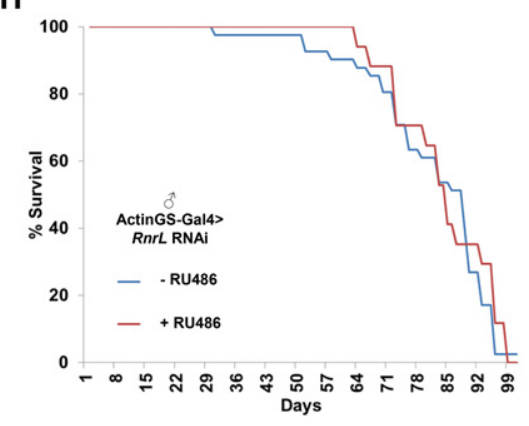

Drosophila S2R cells (Supplemental Fig. 5). To confirm the role of Ahcy13, we performed metabolomic profiling of flies that expressed either Ahcy13 RNAi-1 or control RNAi (against $g f p$ ) under the control of the ubiquitous temperature-sensitive (tubulin-Gal4, tubulin-Gal80ts) driver for $7 \mathrm{~d}$. As expected, Ahcy13 down-regulation caused an $\sim 15$-fold increase in SAH level (Fig. 6B). The knockdown efficacy of Ahcy13 RNAi-1 and Ahcy13 RNAi-2 lines was tested using the ubiquitous Actin5c-Gal4 driver (Supplemental Fig. 3F). Next, to test whether dAhcyLl regulates SAH level, we performed metabolomic profiling of flies expressing dAhcyL1 RNAi-1. In contrast to Ahcy13, down-regulation of dAhcyL1 reduced SAH levels (Fig. 6C) and homocysteine levels (Fig. 6D), the product of SAH hydrolysis, suggesting that down-regulation of dAhcyL1 extends life span by promoting the activity of Ahcyl3 and flux through the methionine cycle. To further test this hypothesis, we analyzed the effect of Ahcyl3 downregulation on life span. RNAi against Ahcy13 using two different RNAi lines using ActinGS at 1 wk of age significantly suppressed life span in males (Ahcy13 RNAi-2, $11.9 \%$ decrease in median life span, $P<0.0001$; and Ahcy13 RNAi-1, 7.5\% decrease in median life span, $P<$ 0.0001) (Fig. 6E,F). In addition, as down-regulation of dAhcyL1 extends life span in a tissue-specific manner (Fig. 4I,K), we tested whether Ahcy13 had the opposite effect on life span. Strikingly, Ahcy13 RNAi-1 expression in the nervous system significantly suppressed life span in males $(10 \%$ median life span decrease, $P<0.0001)$ (Fig. 6G). To further dissect which metabolites upstream of Ahcy 13 are responsible for life span extension, we fed flies with either SAM or a methionine analog that acts as a specific inhibitor of Sam-S (Sufrin et al. 1979). Addition of cycloleucine (Sam-S inhibition) phenocopied methionine depletion and resulted in life span extension $(15.9 \%$ increase in median life span, $P<0.0001$ ), while addition of SAM caused life span suppression $(21 \%$ decrease in median life span with $500 \mu M$ SAM, $P<0.0001$ ) (Fig. $6 \mathrm{H}$, I). Because cycloleucine specifically reduces SAM and SAH levels but not methionine, it indicates that SAM and SAH, but not methionine, are essential for life span extension.

\section{dAhcyL1 and $d A h c y L 2$ are involved in the regulation of H3K4me3 levels and heterochromatin}

The status of methionine metabolism is sufficient to determine H3K4me3 levels (but not H3K9me3 or H3K27me3) in human cells, and H3K4me3 peaks are associated with genes involved in methionine metabolism, establishing a circuit between methionine availability 
A

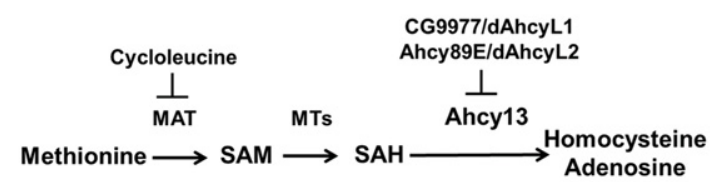

B

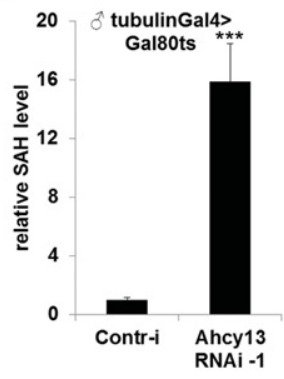

E

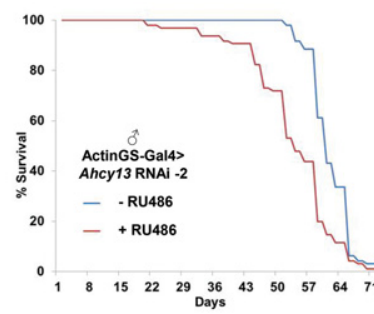

H

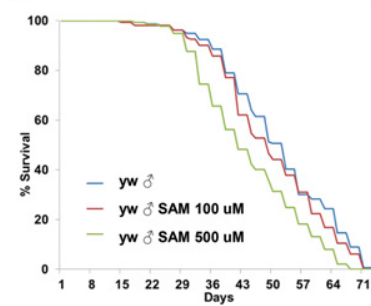

C

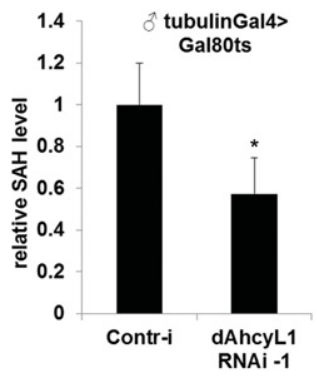

$\mathbf{F}$

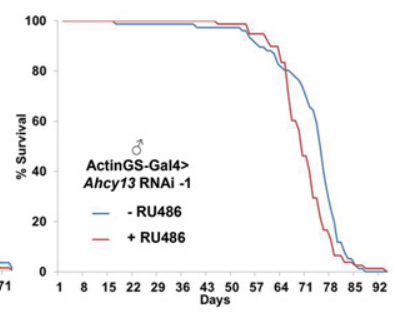

I

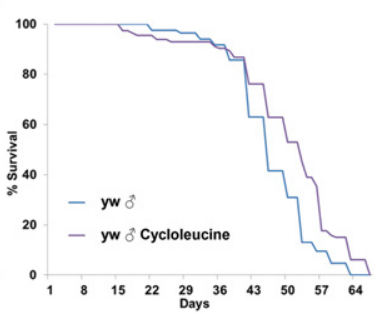

D

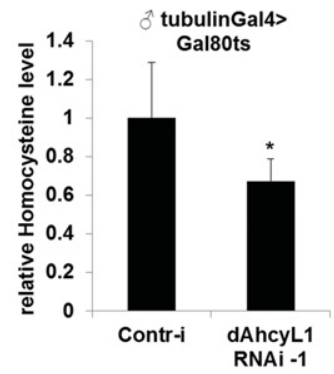

G

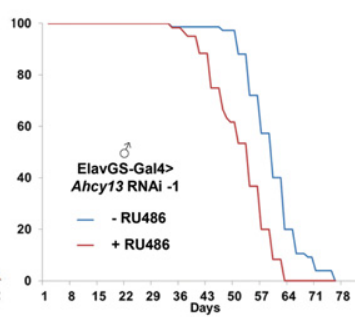

Figure 6. dAhcyL1 and dAhcyL2 are involved in the regulation of methionine metabolism. (A) Scheme of methionine metabolism regulation by dAhcyL1 and dAhcyL2. (B) Relative levels of SAH in tubulinGal80ts, tubulinGal4 flies expressing either control $(g f p)$ or Ahcy13 RNAi-1 for 2 wk at $29.5^{\circ} \mathrm{C}$. $\left.{ }^{* * *}\right) P<0.0001$. Means $\pm \mathrm{SD}$. $(C)$ Relative levels of SAH in tubulinGal80ts, tubulinGal4 flies expressing either control $(g f p)$ or $d A h c y L 1$ RNAi-1 for 2 wk at $\left.29.5^{\circ} \mathrm{C} .{ }^{*}\right) P<0.05$. Means \pm SD. $(D)$ Relative levels of homocysteine in tubulinGal80ts, tubulinGal4 flies expressing either control ( $g f p$ ) or dAhcyL1 RNAi-1 for 2 wk at $29.5^{\circ} \mathrm{C}$. $\left({ }^{*}\right) P<0.05$. Means \pm SD . (E) Ubiquitous adult-onset expression of Ahcy13 RNAi-2 significantly suppresses life span in males. $P<0.001$. (F) Ubiquitous adult-onset expression of Ahcy13 RNAi-1 significantly suppresses life span in males. $P<0.001$. (G) Brain-specific adult-onset expression of Ahcy13 RNAi-1 significantly suppresses life span in males. $P<0.001 .(H)$ Feeding adult flies with SAM significantly suppresses life span in males. $P<0.001$. (I) Feeding adult flies with $100 \mu \mathrm{M}$ cycloleucine significantly increases life span in males. $P<0.001$.

and expression of enzymes of methionine metabolism (Mentch et al. 2015). Thus, we hypothesized that the down-regulation of $d A h c y L 1$ would phenocopy methionine starvation and regulate $\mathrm{H} 3 \mathrm{~K} 4 \mathrm{me} 3$ levels as well as the expression of enzymes involved in methionine metabolism. Strikingly, dAhcyL1 RNAi-1 from 7 to $40 \mathrm{~d}$ significantly suppressed the level of H3K4me3 but not total H3 levels (Fig. 7A). Moreover, dAhcyL1 RNAi- 1 from 7 to $40 \mathrm{~d}$ was associated with a decrease in the expression of three enzymes (ENOPH1, SMS, and MTAP) involved in methionine metabolism (Fig. 7B). These changes suggest a feedback between dAhcyL1 down-regulation and the expression of enzymes involved in methionine metabolism (similar to methionine restriction in mammalian cells) (Mentch et al. 2015).

Aging associated with loss of repressive heterochromatin marks can be measured using a position effect-variegated LacZ reporter gene (Jiang et al. 2013), as the LacZ gene is positioned at the boundary between euchromatin and heterochromatin, and the level of heterochromatization determines the activity of $L a c Z$ expression. To test whether down-regulation of $d A h c y L 1$ affects the level of heterochromatinization, we used ActinGS to express dAhcyL1 RNAi-1 in the presence of different LacZ reporters inserted in either euchromatic or heterochromatic regions. Interestingly, although down-regulation of dAhcyL1 suppressed H3K4me3 levels, down-regulation of $d A h c y L 1$ did not affect the expression of the $\mathrm{LacZ}$ reporter inserted in the heterochromatin (data not shown).

DR extends the life span of many animals, including Drosophila (Tatar et al. 2014). In mice, gene expression profiles of methionine-restricted and calorie-restricted mice do not significantly overlap, suggesting that these two dietary regimens may affect longevity through partly independent pathways (Sun et al. 2009). To test the possible interaction between DR and dAhcyL1 down-regulation in Drosophila, we expressed dAhcyL1 RNAi-1 using the inducible ActinGS and maintained flies with either a standard sugar/yeast diet $(1 \times \mathrm{SY})$ or a diet in which amounts of sugar and yeast were reduced $(0.5 \times \mathrm{SY})$. Both DR $(28 \%$ median life span increase, $P<0.0001)$ and 
A

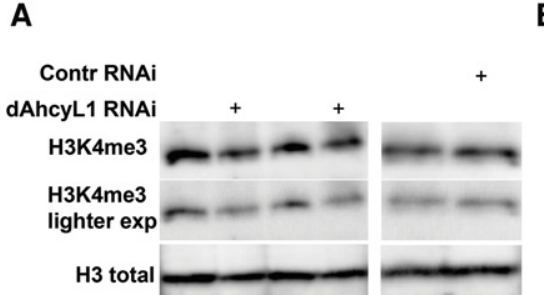

C
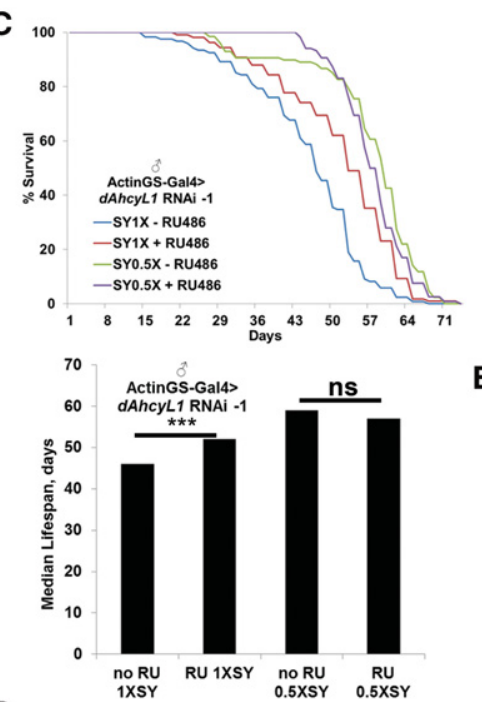

D

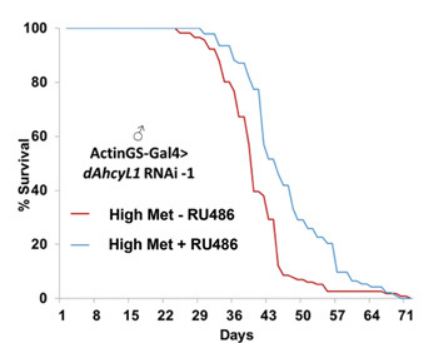

B
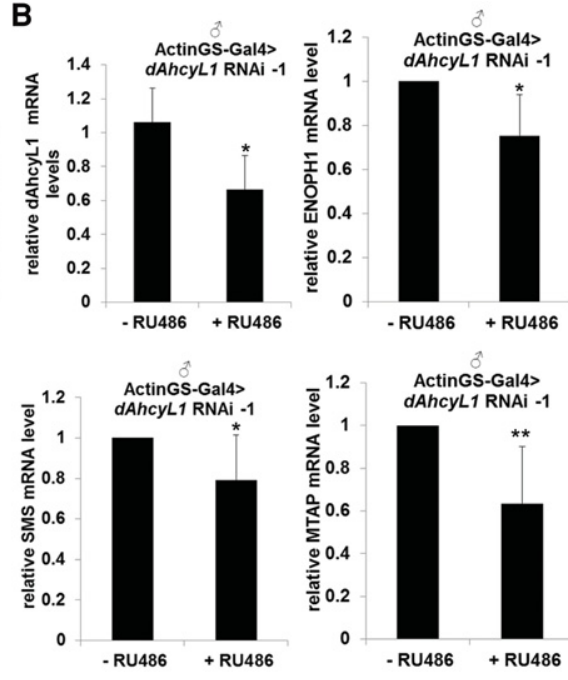

E

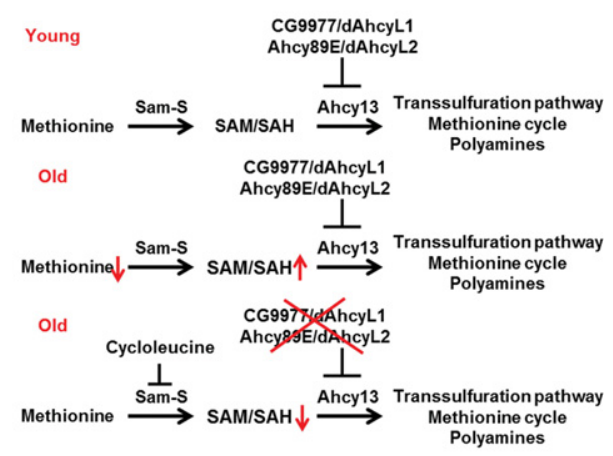

Figure 7. dAhcyL1 and dAhcyL2 are involved in the regulation of $\mathrm{H} 3 \mathrm{~K} 4 \mathrm{me} 3 \mathrm{lev}$ els. (A) Immunoblot analysis of H3K4me3 and total histone 3 in ActinGS-Gal4>dAhcyL1 RNAi-1 or control RNAi flies fed with RU486 from 7 to $40 \mathrm{~d}$ of age. (B) Relative mRNA levels of dAhcyL1, ENOPH1, $S M S$, and MTAP from 40-d-old ActinGSGal4>dAhcyL1 RNAi-1 flies fed with RU486 from 7 to $40 \mathrm{~d}$ of age. (*) $P<0.05$; $(* *) P<0.01$. Means \pm SD. (C) Ubiquitous adult-onset expression of $d A h c y L 1$ RNAi1 increases life span in males under a standard sugar/yeast diet $(1 \times \mathrm{SY}) P<0.001$ but not with a diet where amounts of sugar and yeast were reduced $(0.5 \times S Y)$. (D) Ubiquitous adult-onset expression of dAhcyL1 RNAi-1 increases life span in males maintained on a high-methionine $\operatorname{diet}(4.5 \mathrm{~g} / \mathrm{L})$. (E) Working model. down-regulation of dAhcyL1 (dAhcyL1 RNAi-1, 13\% median life span increase, $P<0.0001)$ significantly extended the life span, but no additional increase was observed when they were combined (Fig. 7C), suggesting that they act through partially overlapping mechanisms. To test the possible interaction between high levels of methionine and $d A h c y L 1$ down-regulation in Drosophila life span, we expressed dAhcyL1 RNAi-1 using the inducible ActinGS and maintained flies on a highmethionine diet $(4.5 \mathrm{~g} / \mathrm{L})$. As expected, the life span of flies was significantly decreased $147 \%$ median life span decrease, $P<0.0001)$. However, down-regulation of dAhcyL1 (dAhcyL1 RNAi-1, 15\% median life span increase, $P<0.0001$ ) significantly extended the life span (Fig. 7D). Overall, our results suggest that higher levels of methionine are not associated with decreased life span because naturally selected $\mathrm{O} 1$ and $\mathrm{O} 3$ longlived flies have higher levels of methionine compared with control B3 flies. Instead, accumulation of the methionine downstream product SAH is detrimental, with its levels being higher in old flies and lower in long-lived flies compared with control flies of the same age. Thus, our results suggest that enhancing flux downstream from SAH via the down-regulation of $d A h c y L 1$ and $d A h c y L 2$ decreases the level of SAH and extends life span (Fig. 7E).

\section{Discussion}

By studying metabolic changes during fly aging, we identified two potential targets, CG9977/dAhcyL1 and Ahcy89E/CG8956/dAhcyL2, for health and life span extension. Whole-body and tissue-specific down-regulation of these two noncanonical, dominant-negative Drosophila homologs of Ahcy (AHCY is the rate-limiting enzyme in methionine metabolism that hydrolyzes SAH to adenosine and homocysteine) significantly extended life span, decreased levels of $\mathrm{SAH}$, and suppressed H3K4me3. 


\section{Age-dependent metabolic reprogramming}

We searched for fly metabolome changes caused by aging and hypothesized that preventing some of these changes would increase life span and prevent agedependent health deterioration. Metabolite profiling revealed striking changes in the metabolome of aged flies, including altered levels of multiple methionine metabolism intermediates as well as several other previously known pathways affected by aging, including glutamate metabolism, glutathione metabolism, and the mitochondrial electron transport chain (Orr et al. 2005; Copeland et al. 2009).

\section{Methionine restriction and life span extension}

Restriction of a single amino acid, either methionine or tryptophan, extends life span in rodents (De Marte and Enesco 1986; Miller et al. 2005). In addition, methionine restriction extends life span in yeast, flies, rodents, and human diploid fibroblasts (Orentreich et al. 1993; Koziel et al. 2014; Lee et al. 2014). Methionine metabolism consists of three branches: salvage, de novo, and transsulfuration pathways. Methionine is converted into glutathione and taurine via the transsulfuration pathway supplying cells with antioxidant defense. In accordance, overexpression of CBS, the rate-limiting enzyme in the transsulfuration pathway, extends life span (Kabil et al. 2011). Moreover, CBS is one of the primary sources of hydrogen sulfide production, which has been shown to function as an evolutionarily conserved mediator of DR-mediated longevity (Hine et al. 2015).

We identified two novel members of methionine metabolism (located upstream of the transsulfuration pathway) that can extend life span when down-regulated. Both dAhcyL1/CG9977 and dAhcyL2/Ahcy89E/CG8956 encode noncanonical AHCY/SAHH enzymes that most likely suppress the function of the canonical AHCY enzyme. A possible explanation for their effects on life span extension is that down-regulation of dAhcyL1 and dAhcyL2 would enhance flux into the transsulfuration pathway. However, we found that cycloleucine, an upstream inhibitor of Sam-S, also increases life span, suggesting that life span extension is most likely due to the clearance of metabolites between Sam-S and Ahcy13 (SAM and SAH).

Gnmt catalyzes the conversion of glycine to sarcosine using SAM as a donor of the methyl group, and recent studies have shown that Gnmt overexpression decreases levels of SAM and extends life span in flies (Obata and Miura 2015). Although Gnmt overexpression extends life span, its product, sarcosine, was identified as a metabolite contributing to prostate cancer progression (Sreekumar et al. 2009), and high cytoplasmic GNMT expression in patient tumor samples correlated with more aggressive forms of prostate cancer (Song et al. 2011). Based on these data, dAhcyL1 and dAhcyL2 could represent better targets for developing methionine restriction mimetics, as they affect methionine indirectly via regulation of $\mathrm{AHCY}$ activity.
Canonical and noncanonical AHCY functions in life span regulation

AHCY (Ahcy13 in flies) is a tetrameric enzyme that catalyzes the reversible hydrolysis of SAH to adenosine and L-homocysteine. SAH is formed as a by-product of SAM through methylation reactions, and hydrolysis of SAH is required to maintain proper concentrations of $\mathrm{SAH}$, which serves as an inhibitor of SAM-dependent methylation reactions. Accordingly, inhibition of AHCY, which is associated with decreased life span, results in the intracellular accumulation of SAH /whole-body adult-onset Ahcy13 RNAi expression caused an $\sim 15$-fold increase in whole-body SAH level). In contrast, down-regulation of dAhcyL1 moderately suppressed levels of SAH (approximately twofold decrease) and increased life span. Interestingly, the level of SAH was increased with age in OreR flies and was significantly lower in naturally selected long-lived flies compared with control flies at $7 \mathrm{wk}$ of age. The mechanisms underlying the age-dependent changes of SAH and which of them affect the agedependent changes in Ahcy13 activity and methionine pathway activity are unknown. Possibly, age-dependent increased oxidative stress can redirect methionine flux into the transsulfuration pathway for glutathione production. It is also worth noting that alterations in SAH levels do not equally affect the activity of methyltransferases (Brosnan and Brosnan 2006), an observation that warrants further investigation.

dAHCYL1/dAHCYL2 proteins consist of a C-terminal AHCY domain and an N-terminal IRBIT domain (Devogelaere et al. 2008). Due to the fact that dAHCYL1/ dAHCYL2 proteins likely have lost their enzyme activity, they can suppress Ahcy13 function via heteromultimerization. As the presence of an N-terminal IRBIT domain gives dAHCYL1/dAHCYL2 proteins new functions in $\mathrm{Ca}^{2+}$ signaling, intracellular $\mathrm{pH}$ regulation, and production of deoxyribonucleotides (which are referred as noncanonical functions), we tested which downstream effectors of dAHCYL1/dAHCYL2 proteins are responsible for life span extension. Down-regulation of $d A h c y L 1$ in the whole body did not affect the levels of deoxyribonucleotides, and the down-regulation of RnrL and Itp-r83A or overexpression of Itp-r83A had no effect on life span. Furthermore, dAhcyL1 down-regulation suppressed the levels of SAH and increased life span, contrary to Ahcy13 function. Altogether, these results suggest that down-regulation of dAhcyL1 promotes life span via modulating Ahcy13 function but not through noncanonical functions. The modular structure of AHCYL proteins suggests that they integrate different signals from $\mathrm{Ca}^{2+}$ signaling, $\mathrm{pH}$ regulation, production of deoxyribonucleotides, SAH clearance, and life span regulation.

\section{Maintenance of euchromatin/heterochromatin states}

Several studies have shown that specific heterochromatin regions are remodeled during aging and that life spanextending interventions such as calorie restriction 
suppress age-dependent heterochromatin remodeling (Wood et al. 2010; Jiang et al. 2013). Moreover, heterochromatin formation prolongs life span, and its status depends on the levels of HP1 and H3K9me (Larson et al. 2012). As the status of methionine metabolism is sufficient to determine the H3K4me3 (but not H3K9me3 or H3K27me3) levels in human cells (Mentch et al. 2015), we hypothesized that down-regulation of dAhcyL1 and dAhcyL2 would mimic methionine starvation and affect H3K4me3 levels and the level of heterochromatinization. Accordingly, we found that down-regulation of dAhcyL1 suppressed H3K4me3 levels. In contrast, we did not observe any effect of down-regulation of dAhcyL1 on expression of a $\mathrm{LacZ}$ reporter gene located in the heterochromatin, which could be explained by differences in the effects of calorie restriction and methionine starvation.

\section{Relevance to cancer, stem cells, and potential therapeutic applications}

Interestingly, the mammalian homolog of Ahcy13 (SAHH) was recovered in a loss-of-function genetic screen as a putative tumor suppressor gene, and its mRNA was lost in $50 \%$ of tumor tissues studied in comparison with normal tissue (Leal et al. 2008). Moreover, elevated homocysteine levels have been reported as a risk factor for dementia and Alzheimer's disease (Seshadri et al. 2002). As we have proposed that down-regulation of dAhcyL1 and dAhcyL2 activates Ahcy13 (SAHH), it will be important to examine whether dAhcyL1/2 can affect carcinogenesis. In addition, methionine metabolism and SAM levels have been shown to be critical for the maintenance and differentiation of human embryonic stem cells (ESCs) and induced pluripotent stem cells (iPSCs) (Shiraki et al. 2014). Interestingly, wild-type flies exhibit age-dependent intestine stem cell (ISC) hyperproliferation and misdifferentiation, causing loss of intestinal integrity, whereas genetic manipulations that improve proliferative homeostasis extend life span (Biteau et al. 2010). Our data suggest that down-regulation of dAhcyL1 and dAhcyL2 suppresses age-dependent $\mathrm{SAH}$ accumulation and prevents loss of intestinal integrity (as revealed by the "Smurf" assay) and that their ubiquitous and tissue-specific down-regulation extends life span. The precise mechanisms of ISC regulation by methionine metabolism and $\mathrm{SAH}$ are interesting subjects for further studies.

\section{Materials and methods}

\section{Life span analysis}

For survival analysis, flies were collected within $24 \mathrm{~h}$ of eclosion, sorted by sex under light $\mathrm{CO}_{2}$ anesthesia, and reared at a standard density (20-25 flies per vial) on cornmeal/soy flour/yeast fly food at $25^{\circ} \mathrm{C}$ and $60 \%$ humidity with a 12 -h on/off light cycle. Flies were transferred to fresh vials every $2 \mathrm{~d}$, and dead flies were counted. RU486 dissolved in ethanol was administered in the medium at the final concentration of $150 \mu \mathrm{g} / \mathrm{mL}$. dAhcyL1 corresponds to CG9977, and $d A h c y L 2$ corresponds to
CG8956/Ahcy89E. The following RNAi lines were used: dAhcyL1 RNAi-1 (HM05009), dAhcyL1 RNAi-2 (HMC04803), dAhcyL2 RNAi-1 (HMJ23469), dAhcyL2 RNAi-2 (8956R-1), Rheb RNAi (HMS00923), w RNAi (HMS00017), Itp-r83A RNAi (HMC03351), gfp RNAi (HMS00314), RnrL RNAi (HMC02351), and Ahcy13 RNAi-1 (HMC03222).

\section{Statistical analysis}

Statistical analyses were performed in either JMP (SAS) or Excel.

\section{Metabolite profiling}

Twenty flies per sample (five biological replicates) were collected, and intracellular metabolites were extracted using $80 \%(\mathrm{v} / \mathrm{v})$ aqueous methanol. A 5500 QTRAP hybrid triple quadrupole mass spectrometer (AB/SCIEX) coupled to a Prominence UFLC high-performance LC (HPLC) system (Shimadzu) was used for steady-state analyses of the samples. Selected reaction monitoring (SRM) of 287 polar metabolites using positive/negative switching with hydrophilic interaction LC (HILIC) was performed. Peak areas from the total ion current for each metabolite SRM transition were integrated using MultiQuant version 2.1 software (AB/SCIEX). The resulting raw data from the MultiQuant software were analyzed using MetaboAnalyst (http://www. metaboanalyst.ca/MetaboAnalyst).

An extended Material and Methods section is in the Supplemental Material.

\section{Acknowledgments}

We thank Trudy Mackay for providing the B3, O1, and O3 flies; John Tower for the GeneSwitch fly stocks; Scott Pletcher for providing the TIGS-2 GeneSwitch driver line; and the Transgenic RNAi Project (TRiP) at Harvard Medical School, supported by National Institutes of Health/National Institute of General Medical Sciences R01-GM084947, for providing transgenic RNAi lines. We thank Mary Salim, Audrey Miller, and Amy Housden for the help with flipping flies, and Min Yuan and Susanne Breitkopf for help with MS experiments. This work was supported by The LAM Foundation Fellowship Award (LAM00105E01-15 to A. A.P.), National Institutes of Health 5P01CA120964-04 (to J.M.A. and N.P.), and National Institutes of Health Dana Farber/Harvard Cancer Center Support Grant P30CA006516-46 (to J.M.A.). F.D. is supported by the Ellison Medical Foundation, the American Federation for Aging Research, and the Glenn Foundation. N.P. is an investigator of the Howard Hughes Medical Institute. A.A. P., R.B., and N.Z. conducted the experiments; A.A.P., J.M.A., F. D., and N.P. designed the experiments; and A.A.P. and N.P. wrote the paper.

\section{References}

Ando H, Mizutani A, Kiefer H, Tsuzurugi D, Michikawa T, Mikoshiba K. 2006. IRBIT suppresses IP3 receptor activity by competing with IP3 for the common binding site on the IP3 receptor. Mol Cell 22: 795-806.

Arnaoutov A, Dasso M. 2014. Enzyme regulation. IRBIT is a novel regulator of ribonucleotide reductase in higher eukaryotes. Science 345: 1512-1515.

Avanesov AS, Ma S, Pierce KA, Yim SH, Lee BC, Clish CB, Gladyshev VN. 2014. Age- and diet-associated metabolome 
remodeling characterizes the aging process driven by damage accumulation. eLife 3: e02077.

Biteau B, Karpac J, Supoyo S, Degennaro M, Lehmann R, Jasper H. 2010. Lifespan extension by preserving proliferative homeostasis in Drosophila. PLoS Genet 6: e1001159.

Bjedov I, Toivonen JM, Kerr F, Slack C, Jacobson J, Foley A, Partridge L. 2010. Mechanisms of life span extension by rapamycin in the fruit fly Drosophila melanogaster. Cell Metab 11: $35-46$.

Brosnan JT, Brosnan ME. 2006. The sulfur-containing amino acids: an overview. J Nutr 136: 1636S-1640S.

Carnes MU, Campbell T, Huang W, Butler DG, Carbone MA, Duncan LH, Harbajan SV, King EM, Peterson KR, Weitzel A, et al. 2015. The genomic basis of postponed senescence in Drosophila melanogaster. PLoS One 10: e0138569.

Clancy DJ, Gems D, Harshman LG, Oldham S, Stocker H, Hafen E, Leevers SJ, Partridge L. 2001. Extension of life-span by loss of CHICO, a Drosophila insulin receptor substrate protein. Science 292: 104-106.

Copeland JM, Cho J, Lo T Jr, Hur JH, Bahadorani S, Arabyan T, Rabie J, Soh J, Walker DW. 2009. Extension of Drosophila life span by RNAi of the mitochondrial respiratory chain. Curr Biol 19: 1591-1598.

De Marte ML, Enesco HE. 1986. Influence of low tryptophan diet on survival and organ growth in mice. Mech Ageing Dev 36: 161-171.

Demontis F, Perrimon N. 2010. FOXO/4E-BP signaling in Drosophila muscles regulates organism-wide proteostasis during aging. Cell 143: 813-825.

Demontis F, Piccirillo R, Goldberg AL, Perrimon N. 2013. Mechanisms of skeletal muscle aging: insights from Drosophila and mammalian models. Dis Model Mech 6: 1339-1352.

Demontis F, Patel VK, Swindell WR, Perrimon N. 2014. Intertissue control of the nucleolus via a myokine-dependent longevity pathway. Cell Rep 7: 1481-1494.

Devogelaere B, Sammels E, De Smedt H. 2008. The IRBIT domain adds new functions to the AHCY family. Bioessays 30: 642-652.

Finkel T. 2015. The metabolic regulation of aging. Nat Med 21: 1416-1423.

Fridell YW, Sanchez-Blanco A, Silvia BA, Helfand SL. 2005. Targeted expression of the human uncoupling protein 2 (hUCP2) to adult neurons extends life span in the fly. Cell Metab 1: 145-152.

Fuchs S, Bundy JG, Davies SK, Viney JM, Swire JS, Leroi AM. 2010. A metabolic signature of long life in Caenorhabditis elegans. BMC Biol 8: 14 .

Giannakou ME, Goss M, Jacobson J, Vinti G, Leevers SJ, Partridge L. 2007. Dynamics of the action of dFOXO on adult mortality in Drosophila. Aging Cell 6: 429-438.

Hine C, Harputlugil E, Zhang Y, Ruckenstuhl C, Lee BC, Brace L, Longchamp A, Trevino-Villarreal JH, Mejia P, Ozaki CK, et al. 2015. Endogenous hydrogen sulfide production is essential for dietary restriction benefits. Cell 160: 132-144.

Hoffman JM, Soltow QA, Li S, Sidik A, Jones DP, Promislow DE. 2014. Effects of age, sex, and genotype on high-sensitivity metabolomic profiles in the fruit fly, Drosophila melanogaster. Aging Cell 13: 596-604.

Jiang N, Du G, Tobias E, Wood JG, Whitaker R, Neretti N, Helfand SL. 2013. Dietary and genetic effects on age-related loss of gene silencing reveal epigenetic plasticity of chromatin repression during aging. Aging 5: 813-824.

Kabil H, Kabil O, Banerjee R, Harshman LG, Pletcher SD. 2011. Increased transsulfuration mediates longevity and dietary restriction in Drosophila. Proc Natl Acad Sci 108: 1683116836.

Koziel R, Ruckenstuhl C, Albertini E, Neuhaus M, Netzberger C, Bust M, Madeo F, Wiesner RJ, Jansen-Durr P. 2014. Methionine restriction slows down senescence in human diploid fibroblasts. Aging Cell 13: 1038-1048.

Lai CQ, Parnell LD, Lyman RF, Ordovas JM, Mackay TF. 2007. Candidate genes affecting Drosophila life span identified by integrating microarray gene expression analysis and QTL mapping. Mech Ageing Dev 128: 237-249.

Landis GN, Abdueva D, Skvortsov D, Yang J, Rabin BE, Carrick J, Tavare S, Tower J. 2004. Similar gene expression patterns characterize aging and oxidative stress in Drosophila melanogaster. Proc Natl Acad Sci 101: 7663-7668.

Larson K, Yan SJ, Tsurumi A, Liu J, Zhou J, Gaur K, Guo D, Eickbush TH, Li WX. 2012. Heterochromatin formation promotes longevity and represses ribosomal RNA synthesis. PLoS Genet 8: e1002473.

Laye MJ, Tran V, Jones DP, Kapahi P, Promislow DE. 2015. The effects of age and dietary restriction on the tissue-specific metabolome of Drosophila. Aging Cell 14: 797-808.

Leal JF, Ferrer I, Blanco-Aparicio C, Hernandez-Losa J, Ramon YCS, Carnero A, Lleonart ME. 2008. S-adenosylhomocysteine hydrolase downregulation contributes to tumorigenesis. Carcinogenesis 29: 2089-2095.

Lee BC, Kaya A, Ma S, Kim G, Gerashchenko MV, Yim SH, Hu Z, Harshman LG, Gladyshev VN. 2014. Methionine restriction extends lifespan of Drosophila melanogaster under conditions of low amino-acid status. Nat Commun 5: 3592.

Lopez-Otin C, Blasco MA, Partridge L, Serrano M, Kroemer G. 2013. The hallmarks of aging. Cell 153: 1194-1217.

Mentch SI, Mehrmohamadi M, Huang L, Liu X, Gupta D, Mattocks D, Gomez Padilla P, Ables G, Bamman MM, Thalacker-Mercer AE, et al. 2015. Histone methylation dynamics and gene regulation occur through the sensing of one-carbon metabolism. Cell Metab 22: 861-873.

Miller RA, Buehner G, Chang Y, Harper JM, Sigler R, SmithWheelock M. 2005. Methionine-deficient diet extends mouse lifespan, slows immune and lens aging, alters glucose, T4, IGF-I and insulin levels, and increases hepatocyte MIF levels and stress resistance. Aging Cell 4: 119-125.

Mourikis P, Hurlbut GD, Artavanis-Tsakonas S. 2006. Enigma, a mitochondrial protein affecting lifespan and oxidative stress response in Drosophila. Proc Natl Acad Sci 103: 1307-1312.

Obata F, Miura M. 2015. Enhancing S-adenosyl-methionine catabolism extends Drosophila lifespan. Nat Commun 6: 8332.

Orentreich N, Matias JR, DeFelice A, Zimmerman JA. 1993. Low methionine ingestion by rats extends life span. I Nutr 123: 269-274.

Orr WC, Radyuk SN, Prabhudesai L, Toroser D, Benes JJ, Luchak JM, Mockett RJ, Rebrin I, Hubbard JG, Sohal RS. 2005. Overexpression of glutamate-cysteine ligase extends life span in Drosophila melanogaster. J Biol Chem 280: 37331-37338.

Osterwalder T, Yoon KS, White BH, Keshishian H. 2001. A conditional tissue-specific transgene expression system using inducible GAL4. Proc Natl Acad Sci 98: 12596-12601.

Owusu-Ansah E, Song W, Perrimon N. 2013. Muscle mitohormesis promotes longevity via systemic repression of insulin signaling. Cell 155: 699-712.

Pletcher SD, Macdonald SJ, Marguerie R, Certa U, Stearns SC, Goldstein DB, Partridge L. 2002. Genome-wide transcript profiles in aging and calorically restricted Drosophila melanogaster. Curr Biol 12: 712-723. 
Poirier L, Shane A, Zheng J, Seroude L. 2008. Characterization of the Drosophila gene-switch system in aging studies: a cautionary tale. Aging Cell 7: 758-770.

Rera M, Bahadorani S, Cho J, Koehler CL, Ulgherait M, Hur JH, Ansari WS, Lo T Jr., Jones DL, Walker DW. 2011. Modulation of longevity and tissue homeostasis by the Drosophila PGC-1 homolog. Cell Metab 14: 623-634.

Rogina B, Reenan RA, Nilsen SP, Helfand SL. 2000. Extended lifespan conferred by cotransporter gene mutations in Drosophila. Science 290: 2137-2140.

Roman G, Endo K, Zong L, Davis RL. 2001. P[Switch], a system for spatial and temporal control of gene expression in Drosophila melanogaster. Proc Natl Acad Sci 98: 12602-12607.

Seshadri S, Beiser A, Selhub J, Jacques PF, Rosenberg IH, D'Agostino RB, Wilson PW, Wolf PA. 2002. Plasma homocysteine as a risk factor for dementia and Alzheimer's disease. N Engl J Med 346: 476-483.

Sgro CM, Partridge L. 1999. A delayed wave of death from reproduction in Drosophila. Science 286: 2521-2524.

Shen J, Curtis C, Tavare S, Tower J. 2009. A screen of apoptosis and senescence regulatory genes for life span effects when over-expressed in Drosophila. Aging 1: 191-211.

Shiraki N, Shiraki Y, Tsuyama T, Obata F, Miura M, Nagae G, Aburatani H, Kume K, Endo F, Kume S. 2014. Methionine metabolism regulates maintenance and differentiation of human pluripotent stem cells. Cell Metab 19: 780-794.

Song YH, Shiota M, Kuroiwa K, Naito S, Oda Y. 2011. The important role of glycine $\mathrm{N}$-methyltransferase in the carcinogenesis and progression of prostate cancer. Mod Pathol 24: 1272-1280.

Sreekumar A, Poisson LM, Rajendiran TM, Khan AP, Cao Q, Yu J, Laxman B, Mehra R, Lonigro RJ, Li Y, et al. 2009. Metabolomic profiles delineate potential role for sarcosine in prostate cancer progression. Nature 457: 910-914.

Stenesen D, Suh JM, Seo J, Yu K, Lee KS, Kim JS, Min KJ, Graff JM. 2013. Adenosine nucleotide biosynthesis and AMPK regulate adult life span and mediate the longevity benefit of caloric restriction in flies. Cell Metab 17: 101-112.

Sufrin JR, Coulter AW, Talalay P. 1979. Structural and conformational analogues of L-methionine as inhibitors of the enzymatic synthesis of S-adenosyl-L-methionine. IV. Further mono-, bi- and tricyclic amino acids. Mol Pharmacol 15: 661-677.

Sun L, Sadighi Akha AA, Miller RA, Harper JM. 2009. Life-span extension in mice by preweaning food restriction and by methionine restriction in middle age. I Gerontol A Biol Sci Med Sci 64: 711-722.

Tatar M, Post S, Yu K. 2014. Nutrient control of Drosophila longevity. Trends Endocrinol Metab 25: 509-517.

Tomas-Loba A, Bernardes de Jesus B, Mato JM, Blasco MA. 2013. A metabolic signature predicts biological age in mice. Aging Cell 12: 93-101.

Ulgherait M, Rana A, Rera M, Graniel J, Walker DW. 2014. AMPK modulates tissue and organismal aging in a non-cell-autonomous manner. Cell Rep 8: 1767-1780.

Wang MC, Bohmann D, Jasper H. 2003. JNK signaling confers tolerance to oxidative stress and extends lifespan in Drosophila. Dev Cell 5: 811-816.

Wood JG, Hillenmeyer S, Lawrence C, Chang C, Hosier S, Lightfoot W, Mukherjee E, Jiang N, Schorl C, Brodsky AS, et al. 2010. Chromatin remodeling in the aging genome of Drosophila. Aging Cell 9: 971-978.

Yu Z, Zhai G, Singmann P, He Y, Xu T, Prehn C, Romisch-Margl W, Lattka E, Gieger C, Soranzo N, et al. 2012. Human serum metabolic profiles are age dependent. Aging Cell 11: 960-967.

Yuan M, Breitkopf SB, Yang X, Asara JM. 2012. A positive/negative ion-switching, targeted mass spectrometry-based metabolomics platform for bodily fluids, cells, and fresh and fixed tissue. Nat Protoc 7: 872-881. 


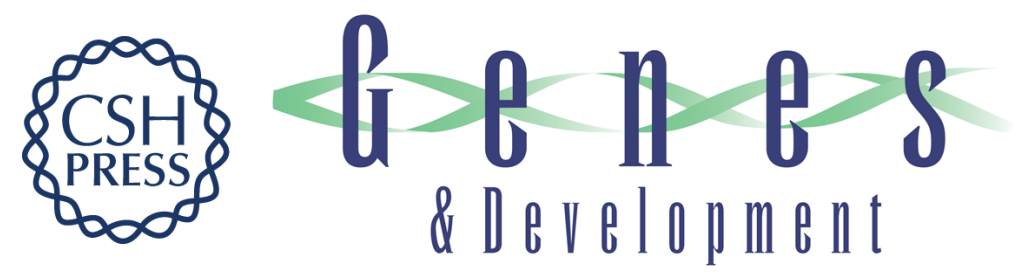

\section{Tissue-specific down-regulation of S-adenosyl-homocysteine via suppression of dAhcyL1/dAhcyL2 extends health span and life span in Drosophila}

Andrey A. Parkhitko, Richard Binari, Nannan Zhang, et al.

Genes Dev. 2016, 30: originally published online June 16, 2016

Access the most recent version at doi:10.1101/gad.282277.116

Supplemental Material

References

Creative

Commons

License

Email Alerting

Service
http://genesdev.cshlp.org/content/suppl/2016/06/16/gad.282277.116.DC1

This article cites 59 articles, 14 of which can be accessed free at: http://genesdev.cshlp.org/content/30/12/1409.full.html\#ref-list-1

This article is distributed exclusively by Cold Spring Harbor Laboratory Press for the first six months after the full-issue publication date (see http://genesdev.cshlp.org/site/misc/terms.xhtml). After six months, it is available under a Creative Commons License (Attribution-NonCommercial 4.0 International), as described at http://creativecommons.org/licenses/by-nc/4.0/.

Receive free email alerts when new articles cite this article - sign up in the box at the top right corner of the article or click here.

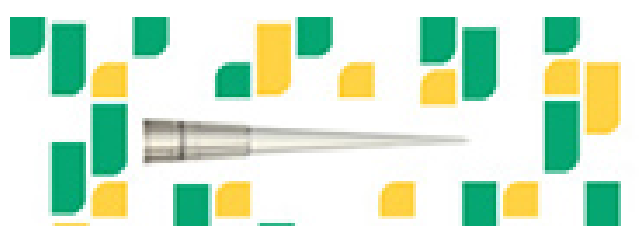

Focused on your science. 